\title{
Avaliação dos níveis de leptina em pacientes submetidos a radioter apia de crânio
}

Autora: Cenata Hosannah Cordeiro de Noronha Pessoa

Orientadora: Professora Marília Matins Guimarães

\section{Resumo}

A leucemia linfoblástica aguda (LLA) é a doença maligna mais comum na infância. Com 0 advento de novos tratamentos quimioterápicos associados ou não à radioterapia de crânio, houve um aumento da sobrevida livre de doença. Tem sido observada uma alta prevalência de obesidade nesses pacientes. Entretanto sua etiologia permanece desconhecida. Trabalhos têm mostrado que tanto a quimioterapia como a radioterapia podem ser responsáveis pela obesidade. A leptina é um hormônio produzido pelo adipócito e encontra-se aumentada nos pacientes obesos. N osso estudo procurou avaliar os níveis de leptina e insulina nesses pacientes. Foram selecionados 26 pacientes (média de idade: $11,48 \pm 4,04$ ) tratados de LLA, sendo 19 pacientes tratados com quimioterapia e radioterapia e 7 apenas com quimioterapia e, comparados com um grupo controle (média de idade: 10,98 2 2,47) semelhante quanto à média do escore $z$ do índice de massa corporal (IMC). A prevalência de obesidade nos nossos pacientes foi significativamente maior do que nos controles ( $36 \% \times 16,67 \%$ ). Encontramos a concentração média de leptina maior nos pacientes do sexo masculino quando comparados aos controles. Houve correlação positiva da leptina com o escore z do IM C. A concentração de leptina correlacionou-se com a de insulina no sexo feminino. A idade do diagnóstico, a dose da radioterapia e o tempo após o final da radioterapia não tiveram influência nos níveis de leptina.

Tese de D outorado apresentada a U niversidade Federal do Rio de Janeiro em 2003. 